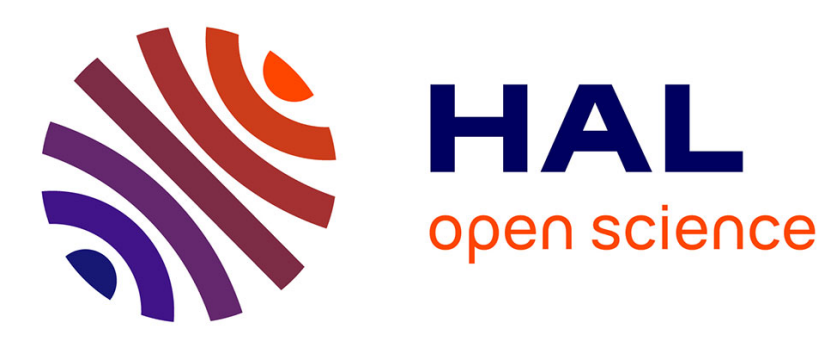

\title{
A model of imperfect interface with damage
}

Elena Bonetti, Giovanna Bonfanti, Frédéric Lebon, Raffaella Rizzoni

\section{To cite this version:}

Elena Bonetti, Giovanna Bonfanti, Frédéric Lebon, Raffaella Rizzoni. A model of imperfect interface with damage. Meccanica, 2017, 52, pp.1911-1922. 10.1007/s11012-016-0520-1 . hal-01694007

\section{HAL Id: hal-01694007 https://hal.science/hal-01694007}

Submitted on 8 Sep 2020

HAL is a multi-disciplinary open access archive for the deposit and dissemination of scientific research documents, whether they are published or not. The documents may come from teaching and research institutions in France or abroad, or from public or private research centers.
L'archive ouverte pluridisciplinaire HAL, est destinée au dépôt et à la diffusion de documents scientifiques de niveau recherche, publiés ou non, émanant des établissements d'enseignement et de recherche français ou étrangers, des laboratoires publics ou privés. 


\title{
A model of imperfect interface with damage
}

\author{
Elena Bonetti · Giovanna Bonfanti • Frédéric Lebon · Raffaella Rizzoni
}

\begin{abstract}
In this paper two models of damaged materials are presented. The first one describes a structure composed by two adherents and an adhesive which is micro-cracked and subject to two different regimes, one in traction and one in compression. The second model is a model of interface derived from the first one through an asymptotic analysis, and it can be interpreted as a model for contact with adhesion and unilateral constraint. Simple numerical examples are presented.
\end{abstract}

Keywords Thin film $\cdot$ Bonding $\cdot$ Asymptotic analysis $\cdot$ Damage $\cdot$ Imperfect interface

\footnotetext{
E. Bonetti

Department of Mathematics, University of Pavia, Pavia, Italy

e-mail: elena.bonetti@unipv.it

G. Bonfanti

Department DICATAM - Section of Mathematics, University of Brescia, Brescia, Italy

e-mail: giovanna.bonfanti@unibs.it

F. Lebon $(\bowtie)$

Laboratory of Mechanics and Acoustics, Aix-Marseille University, CNRS, Centrale Marseille, Marseille, France e-mail: lebon@1ma.cnrs-mrs.fr

R. Rizzoni

Department of Engineering, University of Ferrara, Ferrara, Italy

e-mail: raffaella.rizzoni@unife.it
}

\section{Introduction}

These last years, the study of imperfect interface between solids became a subject of a very large interest for scientists and the industries, in particular because of the development of layered composite materials $[1,9,12,14,17,20,21,31-33,36,37,39-41]$. It is extremely important in particular to control the damage between the fiber and the matrix. Precise models of damaged interface are thus necessary to properly design structures.

In this paper, a model of imperfect interface including damage is proposed. This model is derived by the asymptotic analysis [13, 19, 22, 23, 41, 43, $44,46]$ of a composite structure made of two elastic solids bonded together by a third thin one, which has a non linear behavior. The adhesive substance is microcracked and undergoes a degradation process ruled by two different regimes, one in traction and one in compression. This choice leads to a limit behavior accounting for unilateral condition on the interface. The micro-cracks are modeled using a Kachanov-type model, which has former been applied to cracked composites materials [47], to foamed aluminum [48] and to masonry structures [39].

The paper is divided into three parts. In the first one, the problem of composite body made by three deformable solids bonded together, two adherents and an adhesive is presented. In the second part of the paper, an asymptotic expansion method is applied to 
the problem previously introduced and a model of imperfect interface is derived. In such a model, surface damage effects are included and also impenetrability conditions between the adherents are rendered. Finally, in the last section, the model is applied to a particular cracked material and a simple example in one dimension is studied.

Before proceeding, let us recall some related results in the literature, especially concerning the approach which introduces the interfaces as the limit of a thin medium linking two components. In this frame, we quote the paper [35] where the authors, starting from a structure composed by two adherents and a thin adhesive, obtain a limit model which describes a delamination problem between the adherents and which reflects transmission and impenetrability conditions along the interface. The delamination variable introduced in [35] can be compared to the surface damage parameter used in adhesion models proposed by M. Frémond in [14-16]. Indeed, making use of the phase-field theory, in this approach, the adhesion phenomenon is described in terms of a surface damage parameter related to the state of the micro-bonds between the bodies in contact. The analytical investigation of adhesive contact problems of this type has been addressed in a series of papers: in [2,3] existence, uniqueness and longtime behavior of the solutions have been obtained in the isothermal case, while [4-8] tackled the well-posedness and the longtime analysis of more general models including frictional and thermal effects.

The limit problem obtained in the present paper is very close to the unilateral contact problem with adhesion investigated in [2]. In fact, the variable $l$ which will be introduced to describe the micro-cracks in the thin layer plays the role of the surface damage parameter in adhesive contact problems. More precisely, in [2] the evolution of the adhesion is ruled by a differential inclusion which is strictly related to the equation governing the evolution of the micro-cracks on the interface (the seventh of (31) below). Actually, in [2] also the gradient of the adhesion was included as state variable of the model and a further constraint on the admissible values of the adhesion parameter was imposed.

In this perspective, the asymptotic analysis performed in the present paper can surely represent a further full justification of the adhesive contact models introduced and analyzed in $[2,3]$.

\section{The three-dimensional equations of the composite body}

In the following a composite body made by three deformable solids (see fig. 1), two adherents and an
Fig. 1 Composite body: initial structure and rescaled structure
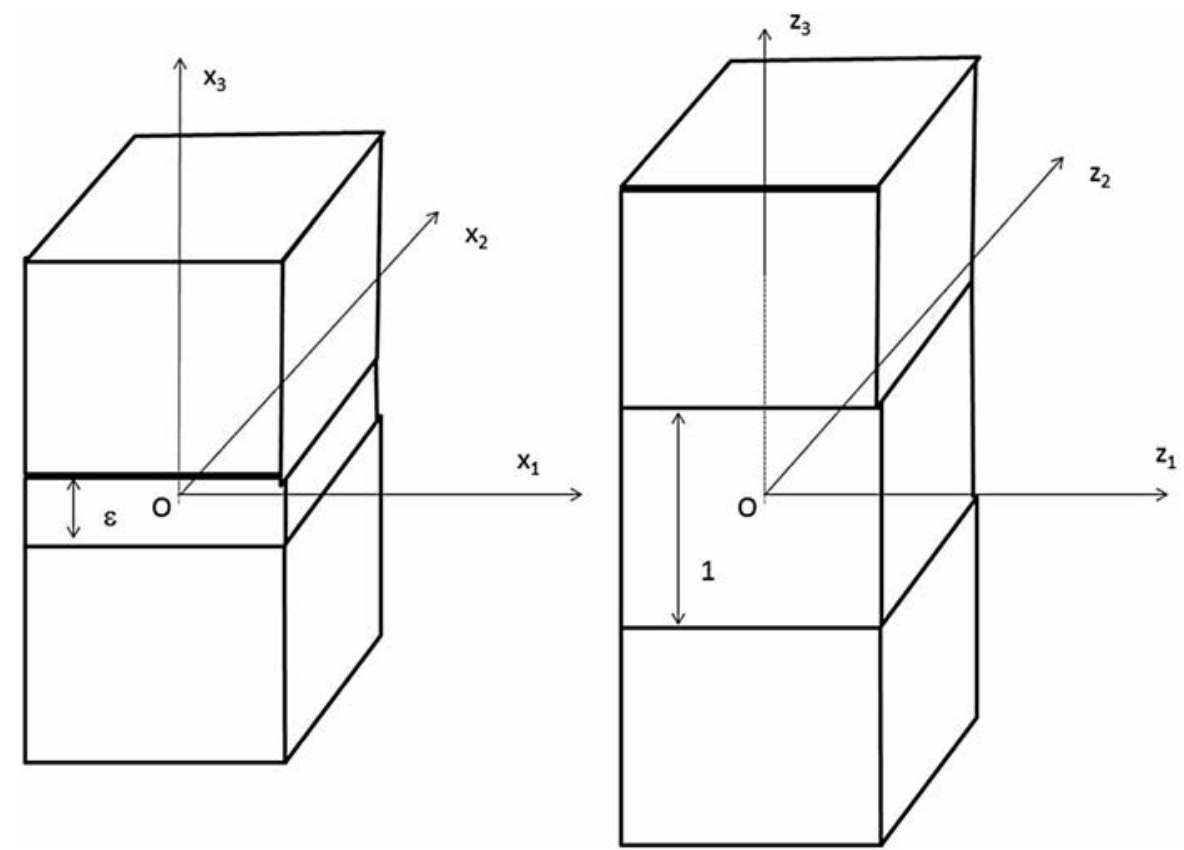
adhesive (also called a glue), and occupying a smooth bounded domain $\Omega^{\varepsilon} \subset \mathrm{IR}^{3}$ is considered. The dependence of the domain $\Omega^{\varepsilon}$ on the parameter $\varepsilon$ will be made precise in the following. An orthonormal Cartesian frame $\left(O, \mathbf{e}_{1}, \mathbf{e}_{2}, \mathbf{e}_{3}\right)$ is introduced and let $\left(x_{1}, x_{2}, x_{3}\right)$ be taken to denote the three coordinates of a particle. The origin lies at the center of the adhesive midplane and the $x_{3}$-axis runs perpendicular to the bounded set $S, S=\left\{\left(x_{1}, x_{2}, x_{3}\right) \in \Omega^{\varepsilon}: x_{3}=0\right\}$ which will be identified in the limit problem as the interface. The adhesive, also called interphase, is occupying the domain $B^{\varepsilon}$, defined by $B^{\varepsilon}=\left\{\left(x_{1}, x_{2}, x_{3}\right) \in \Omega^{\varepsilon}\right.$ : $\left.\left|x_{3}\right|<\frac{\varepsilon}{2}\right\}$. Note that $\varepsilon$ is the thickness of the thin layer $B^{\varepsilon}$. The adherents are occupying respectively the domains $\Omega_{ \pm}^{\varepsilon}$ defined by $\Omega_{ \pm}^{\varepsilon}=\left\{\left(x_{1}, x_{2}, x_{3}\right) \in \Omega^{\varepsilon}\right.$ : $\left.\pm x_{3}>\frac{\varepsilon}{2}\right\}$. The two-dimensional domains $S_{ \pm}^{\varepsilon}$ are taken to denote the interfaces between the adhesive and the adherents, $S_{ \pm}^{\varepsilon}=\left\{\left(x_{1}, x_{2}, x_{3}\right) \in \Omega^{\varepsilon}: x_{3}=\right.$ $\left.\pm \frac{\varepsilon}{2}\right\}$. On a part $S_{g}$ of the boundary $\partial \Omega^{\varepsilon}$, an external load $g$ is applied, and on a part $S_{u}$ of $\partial \Omega^{\varepsilon}$, having strictly positive measure and such that $S_{g} \cap S_{u}=\emptyset$, the displacement is imposed to be equal to 0 . Moreover, it is assumed that the boundary parts $S_{g}$ and $S_{u}$ are located far from the interphase. Finally, a body force $f$ is applied in $\Omega_{ \pm}^{\varepsilon}$, while body forces are neglected in $B^{\varepsilon}$. In the following, $u^{\varepsilon}$ is taken to denote the displacement field, $\sigma^{\varepsilon}$ the Cauchy stress tensor and $e\left(u^{\varepsilon}\right)$ the strain tensor. Under the small strain hypothesis we have $e_{i j}\left(u^{\varepsilon}\right)=\frac{1}{2}\left(u_{i, j}^{\varepsilon}+u_{j, i}^{\varepsilon}\right)$, where as usual notation the comma stands for the partial derivative.

The two adherents are supposed to be elastic, thus

$\sigma_{i j}^{\varepsilon}=a_{i j h k}^{ \pm} e_{h k}\left(u^{\varepsilon}\right)$

coming from the classical constitutive equation

$\sigma^{\varepsilon}=\partial \psi_{, e}^{ \pm}\left(e\left(u^{\varepsilon}\right)\right)$,

where $\psi^{ \pm}=\frac{1}{2} a^{ \pm} e\left(u^{\varepsilon}\right): e\left(u^{\varepsilon}\right)$ is the free energy and $a^{ \pm}$is the fourth order elasticity tensor verifying the usual conditions of positivity and symmetry.

The adhesive is a generalized Kachanov-type material. In the Kachanov theory [34, 49], the constitutive equations are obtained after the homogenization of a micro-cracks material, with $k$ families of cracks. The elastic coefficients depend on the lengths $l_{k}$ of these cracks. In the present paper, only a family of cracks is considered and $l$ is taken the denote the common length of these cracks. Consequently, this parameter can be considered as a damage parameter. Also it is observed that in this theory the stiffness of the material $b^{\varepsilon}(l)$ takes the form $b^{\varepsilon} \approx \varepsilon b(l)$ [39].

As an example, for a crack orthogonal to $\mathbf{e}_{3}$, the Young modulus in the third direction $E_{3}$ is equal to

$$
E_{3}=\frac{E_{0}}{1+2 \rho C E_{0}},
$$

with

$$
C=\frac{\Pi}{2} \frac{1}{\sqrt{E_{0}}}\left(\frac{1}{\mu_{0}}-2 \frac{v_{0}}{E_{0}}+\frac{2}{E_{0}}\right)^{1 / 2}
$$

and $E_{0}$ (resp. $\mu_{0}$, resp. $v_{0}$ ) is the Young modulus (resp. the shear modulus, resp. the Poisson ratio) of the undamaged material and $\rho$ is the density of cracks which is $\rho=\frac{l^{3}}{V}$ in 3 dimensions and $\rho=\frac{l^{2}}{S}$ in 2 dimensions, being $V$ (resp. $S$ ) the volume (resp. the surface) of the representative elementary domain. Note that $V$ and $S$ are proportional to the thickness of the interphase $\varepsilon$.

In order to prevent a possible interpenetration between the adherents, it is supposed that the elasticity coefficients depend totally on the adhesive thickness and crack length in tension, while in compression they depend only partially. In conclusion, in the interphase, two regimes are considered,

$$
\sigma_{i j}^{\varepsilon}= \begin{cases}\varepsilon b_{i j h k}(l) e_{h k}\left(u^{\varepsilon}\right) & \text { if } e^{s}\left(u^{\varepsilon}\right) \geq 0 \\ e^{s}\left(u^{\varepsilon}\right) B_{i j h k}^{\varepsilon}(l) \delta_{h k}+B_{i j h k}^{\varepsilon}(l) e_{h k}^{d}\left(u^{\varepsilon}\right) & \text { if } e^{s}\left(u^{\varepsilon}\right) \leq 0\end{cases}
$$

where $b$ and $B^{\varepsilon}$ are two fourth order elasticity tensors verifying the usual conditions of positivity and symmetry, $e^{s}\left(u^{\varepsilon}\right)=\frac{1}{3} \operatorname{tr}\left(e\left(u^{\varepsilon}\right)\right)\left(\operatorname{resp} . e^{d}\left(u^{\varepsilon}\right)=e\left(u^{\varepsilon}\right)-\right.$ $\left.\frac{1}{3} \operatorname{tr}\left(e\left(u^{\varepsilon}\right)\right) I d\right)$ is the spheric (resp. deviatoric) part of $e\left(u^{\varepsilon}\right)$ and $\delta$ is the Kroenecker symbol.

In addition, the elasticity tensor $B^{\varepsilon}(l)$ verifies the following assumptions which generalize the idea introduced in [35] to anisotropic materials

$$
\left\{\begin{array}{l}
B_{i j h k}^{\varepsilon}(l)=B_{i j h k}^{0}+\varepsilon B_{i j h k}^{1}(l) \text { if }(i, j, h, k) \in I_{l} \\
B_{i j h k}^{\varepsilon}(l)=\varepsilon b_{i j h k}(l) \text { if }(i, j, h, k) \notin I_{l}
\end{array}\right.
$$


with

$I_{l}=\{(1,1,3,3),(2,2,3,3),(3,3,3,3),(1,2,3,3)\}$.

Now, the possible evolution of the crack length $l$ is introduced. Following the general theory proposed in where $\omega^{\varepsilon}$ is a (negative) parameter similar to the Dupré's energy [15, 17] (it plays the role of a cohesion parameter), $l_{0}$ is a given initial crack length and

$$
\psi^{i-s}\left(e\left(u^{\varepsilon}\right), l\right)= \begin{cases}\frac{1}{2} \varepsilon b(l) e\left(u^{\varepsilon}\right): e\left(u^{\varepsilon}\right) & \text { if } e^{s}\left(u^{\varepsilon}\right) \geq 0 \\ \frac{1}{2}\left(e^{s}\left(u^{\varepsilon}\right)\right)^{2} B_{i j h k}^{\varepsilon}(l) \delta_{i j} \delta_{h k}+\frac{1}{2} B_{i j h k}^{\varepsilon}(l) e^{d}\left(u^{\varepsilon}\right): e^{d}\left(u^{\varepsilon}\right) & \text { if } e^{s}\left(u^{\varepsilon}\right) \leq 0\end{cases}
$$

[15], a pseudo-potential of dissipation $\phi$ is considered.

We assume that it is given by the sum of a quadratic term and a positively 1-homogeneous functional, so that the dissipative character will be given by the sum of a rate-dependent and a rate-independent contribution

$\phi(i)=\frac{1}{2} \eta^{\varepsilon} \dot{l}^{2}+I_{[0,+\infty[}(i)$,

where $\eta^{\varepsilon}$ is a positive viscosity parameter and $I_{A}$ denotes the indicator function of the set $A$, i.e. $I_{A}(x)=$ 0 if $x \in A$ and $I_{A}(x)=+\infty$ otherwise. The term $I_{[0,+\infty[}(i)$ forces $i$ to assume non-negative values (i.e. the crack length can not decrease) and renders the irreversible character of the degradation process of the glue.

Here, we are considering a rate-dependent evolution of the damaging process. Note that this choice is compatible with some physical evidence (cf. e.g. $[15,16])$ once we account for viscosity effects and inertia. Actually, some recent papers considered the case of rate-independent evolutions (cf. e.g. [10, 35]). In that cases it can be considered as an activated process and the solution is provided in terms of variational inequality. In the case of rate-dependent evolutions we are able to deal with the equations but at the same time we have to deal with the internal constraint as a suitably defined function.

Moreover, the free energy associated to the constitutive equation of the interphase (eqs. 5) is chosen as follows

$$
\left.\psi^{i}\left(e\left(u^{\varepsilon}\right), l\right)\right)=\psi^{i-s}\left(e\left(u^{\varepsilon}\right), l\right)-\omega^{\varepsilon} l+I_{\left[l_{0},+\infty[\right.}(l)
$$

It is prescribed that $\eta^{\varepsilon}$ and $\omega^{\varepsilon}$ are volumetric densities and thus these two coefficients are inversely proportional to $\varepsilon$. In next sections, we will denote $\eta^{\varepsilon}=\eta / \varepsilon$ and $\omega^{\varepsilon}=\omega / \varepsilon$, with $\eta>0$ and $\omega<0$.

We note that the presence of an indicator function in the free energy (8) renders a physical constraint on the variable $l$. Actually, in the present case, the irreversible character of the damage process guarantees that $l$ cannot decrease and hence, in particular, $l \geq l_{0}$. Thus, we can omit the term $I_{\left[l_{0},+\infty[\right.}(l)$ in (8).

Finally, the fact that the pseudo-potential of dissipation $\phi$ is given by the sum of a quadratic contribution and a 1-homogeneous term and that $\eta_{\varepsilon}>0$ lead to following equation for the damage parameter $l$. We note that the choice of the asymmetric behavior for the strain in tension and compression is similar to the choice introduced in [35] and that it will ensure, in the limit problem, a unilateral constraint on the interface.

$$
\eta^{\varepsilon} i= \begin{cases}\left(\omega^{\varepsilon}-\frac{1}{2} \varepsilon b_{, l}(l) e\left(u^{\varepsilon}\right): e\left(u^{\varepsilon}\right)\right)_{+} & \text {if } e^{s}\left(u^{\varepsilon}\right) \geq 0 \\ \left(\omega^{\varepsilon}-\frac{1}{2} B_{l,}^{\varepsilon}(l) e\left(u^{\varepsilon}\right): e\left(u^{\varepsilon}\right)\right)_{+} & \text {if } e^{s}\left(u^{\varepsilon}\right) \leq 0\end{cases}
$$

where $(\cdot)_{+}$denotes the positive part of a function. Note that $B_{l l}^{\varepsilon}(l)$ is non linearly dependent on $\varepsilon$. In the following $l$ is supposed to be independent of $x_{3}$ i.e. the interphase and the representative volume thicknesses are equal. A more general hypothesis is presented in appendix.

The equilibrium problem of the composite structure is described by the following system 


$$
\left\{\begin{array}{lr}
\sigma_{i j, j}^{\varepsilon}+f_{i}=0 & \text { in } \Omega_{ \pm}^{\varepsilon} \\
\sigma_{i j}^{\varepsilon} n_{j}=g_{i} & \text { on } S_{g} \\
\sigma_{i j, j}^{\varepsilon}=0 & \text { in } B^{\varepsilon} \\
{\left[\left[\sigma_{i 3}^{\varepsilon}\right]\right]=0} & \text { on } S_{ \pm}^{\varepsilon} \\
{\left[\left[u_{i}^{\varepsilon}\right]\right]=0} & \text { on } S_{ \pm}^{\varepsilon} \\
u_{i}^{\varepsilon}=0 & \text { on } S_{u} \\
\sigma_{i j}^{\varepsilon}=a_{i j h k}^{ \pm} e_{h k}\left(u^{\varepsilon}\right) & \text { in } \Omega_{ \pm}^{\varepsilon} \\
\sigma_{i j}^{\varepsilon}=\varepsilon b_{i j h k}(l) e_{h k}\left(u^{\varepsilon}\right) \text { if } e^{s}\left(u^{\varepsilon}\right) \geq 0 & \text { in } B^{\varepsilon} \\
\sigma_{i j}^{\varepsilon}=e^{s}\left(u^{\varepsilon}\right) B_{i j h k}^{\varepsilon}(l) \delta_{h k}+B_{i j h k}^{\varepsilon}(l) e_{h k}^{d}\left(u^{\varepsilon}\right) \text { if } e^{s}\left(u^{\varepsilon}\right) \leq 0 & \text { in } B^{\varepsilon} \\
\eta^{\varepsilon} i=\left(\omega^{\varepsilon}-\frac{1}{2} \varepsilon b_{, l}(l) e\left(u^{\varepsilon}\right): e\left(u^{\varepsilon}\right)\right)_{+} \text {if } e^{s}\left(u^{\varepsilon}\right) \geq 0 & \text { in } B^{\varepsilon} \\
\eta^{\varepsilon} i=\left(\omega^{\varepsilon}-\frac{1}{2} B_{, l}^{\varepsilon}(l) e\left(u^{\varepsilon}\right): e\left(u^{\varepsilon}\right)\right)_{+} \text {if } e^{s}\left(u^{\varepsilon}\right) \leq 0 & \text { in } B^{\varepsilon}
\end{array}\right.
$$

supplemented by a given initial condition $l_{0}$ on the crack length variable $l$.

In (11), $[[f]]$ denotes the jump of $f$ across $S_{ \pm}^{\varepsilon}$ i.e. $f\left(( \pm \varepsilon / 2)^{ \pm}\right)-f\left(( \pm \varepsilon / 2)^{\mp}\right), \quad$ where $\quad f\left(a^{+}\right)=$ $\lim _{x \longrightarrow a, x>a} f(x)$ and $f\left(a^{-}\right)=\lim _{x \longrightarrow a, x<a} f(x)$.

\section{The asymptotic expansion method}

Since the thickness of the interphase is very small, it is natural to seek the solution of problem (11) using asymptotic expansions with respect to the parameter $\varepsilon$ [24-28, 30, 45]. In particular, the following asymptotic series with integer powers are assumed:

$$
\left\{\begin{array}{l}
\mathbf{u}^{\varepsilon}=\mathbf{u}^{0}+\varepsilon \mathbf{u}^{1}+o(\varepsilon) \\
\sigma^{\varepsilon}=\sigma^{0}+\varepsilon \sigma^{1}+o(\varepsilon) .
\end{array}\right.
$$

The domain is then rescaled (see figure 1) using a classical procedure [11] :

- In the adhesive, the following change of variable is introduced

$$
\begin{gathered}
\left(x_{1}, x_{2}, x_{3}\right) \in B^{\varepsilon} \rightarrow\left(z_{1}, z_{2}, z_{3}\right) \in B, \\
\text { with }\left(z_{1}, z_{2}, z_{3}\right)=\left(x_{1}, x_{2}, \frac{x_{3}}{\varepsilon}\right)
\end{gathered}
$$

and it is set $\hat{\mathbf{u}}^{\varepsilon}\left(z_{1}, z_{2}, z_{3}\right)=\mathbf{u}^{\varepsilon}\left(x_{1}, x_{2}, x_{3}\right)$ and $\hat{\sigma}^{\varepsilon}\left(z_{1}, z_{2}, z_{3}\right)=\sigma^{\varepsilon}\left(x_{1}, x_{2}, x_{3}\right), \quad$ where $\quad B=$ $\left\{\left(x_{1}, x_{2}, x_{3}\right) \in \Omega:\left|x_{3}\right|<\frac{1}{2}\right\}$.

- In the adherents, the following change of variable is introduced

$$
\begin{aligned}
& \left(x_{1}, x_{2}, x_{3}\right) \in \Omega_{ \pm}^{\varepsilon} \rightarrow\left(z_{1}, z_{2}, z_{3}\right) \in \Omega_{ \pm} \\
& \quad \text { with }\left(z_{1}, z_{2}, z_{3}\right)=\left(x_{1}, x_{2}, x_{3} \pm 1 / 2 \mp \varepsilon / 2\right)
\end{aligned}
$$

and it is set $\overline{\mathbf{u}}^{\varepsilon}\left(z_{1}, z_{2}, z_{3}\right)=\mathbf{u}^{\varepsilon}\left(x_{1}, x_{2}, x_{3}\right)$ and $\bar{\sigma}^{\varepsilon}\left(z_{1}, z_{2}, z_{3}\right)=\sigma^{\varepsilon}\left(x_{1}, x_{2}, x_{3}\right)$, where $\quad \Omega_{ \pm}=$ $\left\{\left(x_{1}, x_{2}, x_{3}\right) \in \Omega: \pm x_{3}>\frac{1}{2}\right\}$. The external forces are assumed to be independent of $\varepsilon$. As a consequence, it is set $\bar{f}\left(z_{1}, z_{2}, z_{3}\right)=f\left(x_{1}, x_{2}, x_{3}\right)$ and $\bar{g}\left(z_{1}, z_{2}, z_{3}\right)=g\left(x_{1}, x_{2}, x_{3}\right)$.

The governing equations of the rescaled problem are as follows: 


$$
\begin{cases}\bar{\sigma}_{i j, j}^{\varepsilon}+\bar{f}_{i}=0 & \text { in } \Omega_{ \pm} \\ \bar{\sigma}_{i j}^{\varepsilon} n_{j}=\bar{g}_{i} & \text { on } \bar{S}_{g} \\ \hat{\sigma}_{i j, j}^{\varepsilon}=0 & \text { in } B \\ \bar{\sigma}_{i 3}^{\varepsilon}=\hat{\sigma}_{i 3}^{\varepsilon} & \text { on } S_{ \pm} \\ \bar{u}_{i}^{\varepsilon}=\hat{u}_{i}^{\varepsilon} & \text { on } S_{ \pm} \\ \bar{u}_{i}^{\varepsilon}=0 & \text { on } \bar{S}_{u} \\ \bar{\sigma}_{i j}^{\varepsilon}=\bar{a}_{i j h k}^{ \pm} \bar{e}_{h k}\left(\bar{u}^{\varepsilon}\right) & \text { in } \Omega_{ \pm} \\ \hat{\sigma}_{i j}^{\varepsilon}=\varepsilon \hat{b}_{i j h k}(l) \hat{e}_{h k}\left(\hat{u}^{\varepsilon}\right) \text { if } \hat{e}^{s}\left(\hat{u}^{\varepsilon}\right) \geq 0 & \text { in } B \\ \hat{\sigma}_{i j}^{\varepsilon}=\hat{e}^{s}\left(\hat{u}^{\varepsilon}\right) \hat{B}_{i j h k}^{\varepsilon}(l) \delta_{h k}+\hat{B}_{i j h k}^{\varepsilon}(l) \hat{e}_{h k}^{d}\left(\hat{u}^{\varepsilon}\right) \text { if } \hat{e}^{s}\left(\hat{u}^{\varepsilon}\right) \leq 0 & \text { in } B \\ \hat{\eta}^{\varepsilon} \dot{\hat{l}}=\left(\hat{\omega}^{\varepsilon}-\frac{1}{2} \varepsilon \hat{b}_{, l}(l) \hat{e}\left(\hat{u}^{\varepsilon}\right): \hat{e}\left(\hat{u}^{\varepsilon}\right)\right)_{+} \text {if } \hat{e}^{s}\left(\hat{u}^{\varepsilon}\right) \geq 0 & \text { in } B \\ \hat{\eta}^{\varepsilon} \dot{\hat{l}}=\left(\hat{\omega}^{\varepsilon}-\frac{1}{2} \hat{B}_{, l}^{\varepsilon}(l) \hat{e}\left(\hat{u}^{\varepsilon}\right): \hat{e}\left(\hat{u}^{\varepsilon}\right)\right)_{+} \text {if } \hat{e}^{s}\left(\hat{u}^{\varepsilon}\right) \leq 0 & \text { in } B\end{cases}
$$

where $S_{ \pm}=\left\{\left(x_{1}, x_{2}, x_{3}\right) \in \Omega: x_{3}= \pm \frac{1}{2}\right\}$ and $\overrightarrow{.}, \hat{.}$ denote the rescaled operators in the adherents and in the adhesive, respectively.

In view of (12) the displacement and stress fields are written as asymptotic expansions

$$
\left\{\begin{array}{l}
\hat{\sigma}^{\varepsilon}=\hat{\sigma}^{0}+\varepsilon \hat{\sigma}^{1}+o(\varepsilon) \\
\hat{u}^{\varepsilon}=\hat{u}^{0}+\varepsilon \hat{u}^{1}+o(\varepsilon) \\
\bar{\sigma}^{\varepsilon}=\bar{\sigma}^{0}+\varepsilon \bar{\sigma}^{1}+o(\varepsilon) \\
\bar{u}^{\varepsilon}=\bar{u}^{0}+\varepsilon \bar{u}^{1}+o(\varepsilon),
\end{array}\right.
$$

in the rescaled adhesive and adherents, respectively.

\subsection{Expansions of the equilibrium equations} in the adherents

Substituting (14) into the first, second, sixth and seventh equations of (13), it is obtained at the first order of expansion (power 0)

$$
\begin{cases}\bar{\sigma}_{i j, j}^{0}+\bar{f}_{i}=0 & \text { in } \Omega_{ \pm} \\ \bar{\sigma}_{i j}^{0} n_{j}=\bar{g}_{i} & \text { on } \bar{S}_{g} \\ \bar{u}_{i}^{0}=0 & \text { on } \bar{S}_{u} \\ \bar{\sigma}_{i j}^{0}=\bar{a}_{i j h k}^{ \pm} \bar{e}_{h k}\left(\bar{u}^{0}\right) & \text { in } \Omega_{ \pm}\end{cases}
$$

3.2 Expansions of the equilibrium equations in the adhesive

Substituting (14) into the third equation of (13) it is deduced that the following conditions hold in $B$ (power -1):

$\hat{\sigma}_{i 3,3}^{0}=0$,

i.e. $\hat{\sigma}_{i 3}^{0}$ does not depend on $z_{3}$, and it can be expressed as

$$
\left[\hat{\sigma}_{i 3}^{0}\right]=0,
$$

where $[f]=f\left(x_{1}, x_{2}, \frac{1}{2}\right)-f\left(x_{1}, x_{2},-\frac{1}{2}\right)$. In the adhesive the strain field becomes:

$\hat{e}\left(\hat{u}^{\varepsilon}\right)=\varepsilon^{-1} \hat{e}^{-1}+\hat{e}^{0}+\varepsilon \hat{e}^{1}+o(\varepsilon)$

where

$$
\begin{gathered}
\hat{e}_{33}^{-1}=\hat{u}_{3,3}^{0} \\
\hat{e}_{\alpha 3}^{-1}=\frac{1}{2} \hat{u}_{\alpha, 3}^{0}, \alpha=1,2
\end{gathered}
$$

It is obvious to remark that

$\hat{e}^{s}\left(\hat{u}^{\varepsilon}\right)=\frac{1}{3}\left(\varepsilon^{-1} \hat{u}_{3,3}^{0}+\hat{u}_{1,1}^{0}+\hat{u}_{2,2}^{0}+\hat{u}_{3,3}^{1}+o(1)\right)$

and that at the lowest order of expansion the sign of the spherical part of the strain tensor is the sign of $\hat{u}_{3,3}^{0}$. The 
eighth equation in (13) is next considered i.e. $\hat{u}_{3,3}^{0} \geq 0$. At the first order in the expansions (power 0), it is obtained $\hat{\sigma}_{i 3}^{0}=\hat{b}_{i 3 j 3}(l) \hat{u}_{j, 3}^{0}$

It is observed that $\hat{\sigma}_{i 3}^{0}$ (and thus $\hat{u}_{i, 3}^{0}$ ) does not depend on $z_{3}$, thus

$$
\hat{\sigma}_{i 3}^{0}=\hat{b}_{i 3 j 3}(l)\left[\hat{u}_{j}^{0}\right]
$$

which is the classical equation of soft interface. Denoting $\hat{b}_{i 3 j 3}(l)=K_{i j}^{33}(l)$, it is obtained

$$
\hat{\sigma}^{0} \mathbf{e}_{3}=K^{33}(l)\left[\hat{u}^{0}\right] \text { if }\left[\hat{u}_{3}^{0}\right] \geq 0 \text { on } S^{ \pm}
$$

Now the ninth equation in (13) is considered. At the lowest order (power -1 ), the expansion gives

$$
0=\hat{B}_{i i 33}^{0} \hat{u}_{3,3}^{0} \text { if } \hat{u}_{3,3}^{0} \leq 0
$$

or due to the positivity of $\hat{B}^{0}$

$$
\hat{u}_{3,3}^{0}=0 \text { if } \hat{u}_{3,3}^{0} \leq 0
$$

Being $\hat{u}_{3,3}^{0}$ independent of $z_{3}$, it can be deduced that

$$
\left[\hat{u}_{3}^{0}\right]=0 \text { if }\left[\hat{u}_{3}^{0}\right] \leq 0
$$

Note that at the second order in the expansions (power $0)$, it is obtained

$$
\begin{aligned}
\hat{\sigma}_{i 3}^{0}= & \hat{b}_{i 3 j 3}(l) \hat{u}_{j, 3}^{0}, i=1,2 \\
\hat{\sigma}_{33}^{0}= & \hat{B}_{33 \alpha \alpha}^{0} \hat{u}_{\alpha, \alpha}^{0}+\hat{B}_{3333}^{1} \hat{u}_{3,3}^{0}+\hat{B}_{3333}^{0} \hat{u}_{3,3}^{1} \\
& +\hat{b}_{33 j 3}(l) \hat{u}_{j, 3}^{0}, \alpha \in\{1,2\}
\end{aligned}
$$

or

$$
\begin{aligned}
& \hat{\sigma}_{i 3}^{0}=K_{i j}^{33}(l)\left[\hat{u}_{j}^{0}\right] \\
& \hat{\sigma}_{33}^{0}=K_{3 j}^{33}(l)\left[\hat{u}_{j}^{0}\right]+\hat{\tau}^{0}
\end{aligned}
$$

with $\hat{\tau}^{0}=\left[\hat{B}_{33 \alpha \alpha}^{0} \hat{u}_{\alpha, \alpha}^{0}+\hat{B}_{3333}^{0} \hat{u}_{3,3}^{1}\right]$. It is observed that at the second order of expansion the sign of the spherical part of the strain tensor is the sign of $\hat{u}_{\alpha, \alpha}^{0}+\hat{u}_{3,3}^{1}$ and if the material is isotropic, $\hat{\tau}^{0} \leq 0$.

In conclusion, it is obtained on $S^{ \pm}$

$$
\begin{aligned}
& \hat{\sigma}^{0} \mathbf{e}_{3}=K^{33}(l)\left[\hat{u}^{0}\right] \text { if }\left[\hat{u}_{3}^{0}\right] \geq 0 \\
& \hat{\sigma}^{0} \mathbf{e}_{3}=K^{33}(l)\left[\hat{u}^{0}\right]+\hat{\tau}^{0} \mathbf{e}_{3},\left[\hat{u}_{3}^{0}\right]=0 \text { if }\left[\hat{u}_{3}^{0}\right] \leq 0
\end{aligned}
$$

Now the two last equations in (13) are considered. If we consider that the length $l$ cannot decrease , the first term in the expansion gives (power -1)

$$
\begin{aligned}
\hat{\eta} \hat{\hat{l}} & =\hat{\omega}-\frac{1}{2}\left(\hat{b}_{, l}(l)\right)_{i 3 j 3} \hat{u}_{j, 3}^{0}: \hat{u}_{i, 3}^{0} \\
& =\hat{\omega}-\frac{1}{2}\left(K_{, l}^{33}(l)\right)_{i j} \hat{u}_{j, 3}^{0} \cdot \hat{u}_{i, 3}^{0}
\end{aligned}
$$

This equation can be integrated along the third direction in two steps, considering that $\hat{\sigma}_{i 3}^{0}=$ $K_{i j}^{33}(l) \hat{u}_{j, 3}^{0}$ or $\hat{u}_{, 3}^{0}=\left(K^{33}(l)\right)^{-1} \hat{\sigma}^{0} \mathbf{e}_{3}$. Thus, it is obtained $\hat{\eta} \hat{\hat{l}}=\hat{\omega}-\frac{1}{2} K_{, l}^{33}(l)\left[\hat{u}^{0}\right] \cdot\left[\hat{u}^{0}\right]$

which can be decomposed, as classical, into normal and tangential parts

$$
\hat{\eta} \hat{\hat{l}}=\hat{\omega}-\frac{1}{2} K_{N, l}^{33}(l)\left[\hat{u}_{N}^{0}\right]^{2}-\frac{1}{2} K_{T, l}^{33}(l)\left[\hat{u}_{T}^{0}\right] \cdot\left[\hat{u}_{T}^{0}\right]
$$

3.3 Matching between the adhesive and the adherents

Substituting (16) into the fourth and fifth equations of (13), it is deduced that the following conditions hold on $S_{ \pm}$:

$$
\begin{aligned}
\hat{\sigma}_{i 3}^{0}\left(z_{1}, z_{2}, \pm \frac{1}{2}\right) & =\bar{\sigma}_{i 3}^{0}\left(z_{1}, z_{2}, \pm \frac{1}{2}\right)=\sigma_{i 3}^{0}\left(x_{1}, x_{2}, \pm \frac{\varepsilon}{2}\right) \\
& \approx \sigma_{i 3}^{0}\left(x_{1}, x_{2}, 0\right) \\
\hat{u}_{i}^{0}\left(z_{1}, z_{2}, \pm \frac{1}{2}\right) & =\bar{u}_{i}^{0}\left(z_{1}, z_{2}, \pm \frac{1}{2}\right)=u_{i}^{0}\left(x_{1}, x_{2}, \pm \frac{\varepsilon}{2}\right) \\
& \approx u_{i}^{0}\left(x_{1}, x_{2}, 0^{ \pm}\right)
\end{aligned}
$$

In conclusion, it is obtained

$$
\begin{cases}\sigma_{i j, j}^{0}+f_{i}=0 & \text { in } \Omega_{ \pm} \\ \sigma_{i j}^{0} n_{j}=g_{i} & \text { on } S_{g} \\ u_{i}^{0}=0 & \text { on } S_{u} \\ \sigma_{i j}^{0}=a_{i j h k}^{ \pm} e_{h k}\left(u^{0}\right) & \text { in } \Omega_{ \pm} \\ \sigma_{i 3}^{0}=K_{i j}^{33}(l)\left[u_{j}^{0}\right]_{+}+\tau^{0} \delta_{i 3} & \text { on } S \\ {\left[u_{3}^{0}\right] \tau^{0}=0} & \text { on } S \\ \eta i=\left(\omega-\frac{1}{2} K_{l,}^{33}(l)\left[u^{0}\right]_{+} \cdot\left[u^{0}\right]_{+}\right)_{+} & \text {on } S\end{cases}
$$


where $\left[u^{0}\right]_{+}=\left[u^{0}\right]$ if $\left[u_{3}^{0}\right] \geq 0,\left[u^{0}\right]_{+}=\left(\left[u_{1}^{0}\right],\left[u_{2}^{0}\right], 0\right)^{T}$ if $\left[u_{3}^{0}\right] \leq 0$.

These equations give a model of imperfect soft interface with unilateral contact and damage evolution. Note that the variable $l$ (length variable) can be compared with the density of adhesion (a dimentionless variable) introduced by M. Frémond in [14]. This intensity of adhesion can be interpreted mechanically as the ratio $l / l_{0}$.

\section{An example in 2D: Kachanov material}

\subsection{Constitutive equations}

In two dimensions ( the plane $\left.\left(O, \mathbf{e}_{1}, \mathbf{e}_{2}\right)\right)$, the system can be rewritten as

$$
\begin{cases}\sigma_{i j, j}^{0}+f_{i}=0 & \text { in } \Omega_{ \pm} \\ \sigma_{i j}^{0} n_{j}=g_{i} & \text { on } S_{g} \\ u_{i}^{0}=0 & \text { on } S_{u} \\ \sigma_{i j}^{0}=a_{i j h k}^{ \pm} e_{h k}\left(u^{0}\right) & \text { in } \Omega_{ \pm} \\ \sigma_{i 2}^{0}=K_{i j}^{22}(l)\left[u_{j}^{0}\right]_{+}+\tau^{0} \delta_{i 2} & \text { on } S \\ {\left[u_{2}^{0}\right] \tau^{0}=0} & \text { on } S \\ \eta \dot{l}=\left(\omega-\frac{1}{2} K_{, l}^{22}(l)\left[u^{0}\right]_{+} \cdot\left[u^{0}\right]_{+}\right)_{+} & \text {on } S\end{cases}
$$

For the Kachanov model of homogenized cracked material [34, 49], the stiffness matrix is written, in engineering notation, as

$$
K^{\varepsilon}=\left[\begin{array}{ccc}
E_{0} & \frac{v_{0} L \varepsilon}{2 l^{2} C} & 0 \\
\frac{v_{0} L \varepsilon}{2 l^{2} C} & \frac{L \varepsilon}{2 l^{2} C} & 0 \\
0 & 0 & \frac{L \varepsilon}{l^{2} C}
\end{array}\right]
$$

where $L$ is the length of the interphase, $C$ is given in (4). It is supposed that the crack is along $x_{1}$ axis. Matrix $K^{33}$ (in fact $K^{22}$ in this configuration), which is diagonal, reads

$$
K^{22}=\left[\begin{array}{cc}
\frac{L}{2 l^{2} C} & 0 \\
0 & \frac{L}{l^{2} C}
\end{array}\right]
$$

and its derivative reads

$$
K_{, l}^{22}=\left[\begin{array}{cc}
\frac{-L}{l^{3} C} & 0 \\
0 & \frac{-2 L}{l^{3} C}
\end{array}\right]
$$

The last equation in (32), providing the evolution of $l$ is written as

$$
\eta \dot{l}=\left(\omega+\frac{L}{2 l^{3} C}\left[u_{1}^{0}\right]^{2}+\frac{L}{l^{3} C}\left[u_{2}^{0}\right]_{+}^{2}\right)_{+}
$$

\subsection{A focus on the crack length evolution}

As a first approach to the evolution of $l$, we can consider the simplified case of vanishing viscosity $\eta=$ 0 and $\left[u_{1}^{0}\right]=0$. Note that in compression one has $\left[u_{2}^{0}\right]=0$ (unilateral contact). In traction, until $\frac{L}{l^{3} C}\left[u_{2}^{0}\right]_{+}^{2} \leq-\omega$ i.e. $\left[u_{2}^{0}\right] \leq \sqrt{-\frac{\omega l^{3} C}{L}}=\delta_{2}$ the stiffness is constant and the relation between $\sigma_{22}$ and $\left[u_{2}^{0}\right]$ is linear, with the slope equal to $\frac{L}{2 l^{2} C}$. We take $\sigma_{22}^{\max }$ to denote $\frac{1}{2} \sqrt{-\frac{\omega L}{C l}}$, the maximum value of $\sigma_{22}$. When the threshold is reached, we have

$\left.l=-\frac{L\left[u_{2}^{0}\right]^{2}}{\omega C}\right)^{1 / 3}$

and then

$\left.\sigma_{22}=\frac{1}{2} \frac{L \omega^{2}}{C\left[u_{2}^{0}\right]}\right)^{1 / 3}$

This relation is represented on fig. 2. On fig. 2, the relation between $\sigma_{22}$ and $\left[u^{0}\right]$ is represented approximatively (using implicit Euler integration schema) for various values of the viscosity.

\subsection{A simple academic example}

In this section, the example of a simple bar of length $L_{0}$ is considered (see fig. 3). The bar is bonded on its left part and loaded on its right part. The volumic force is neglected. The force on the right part is given by 


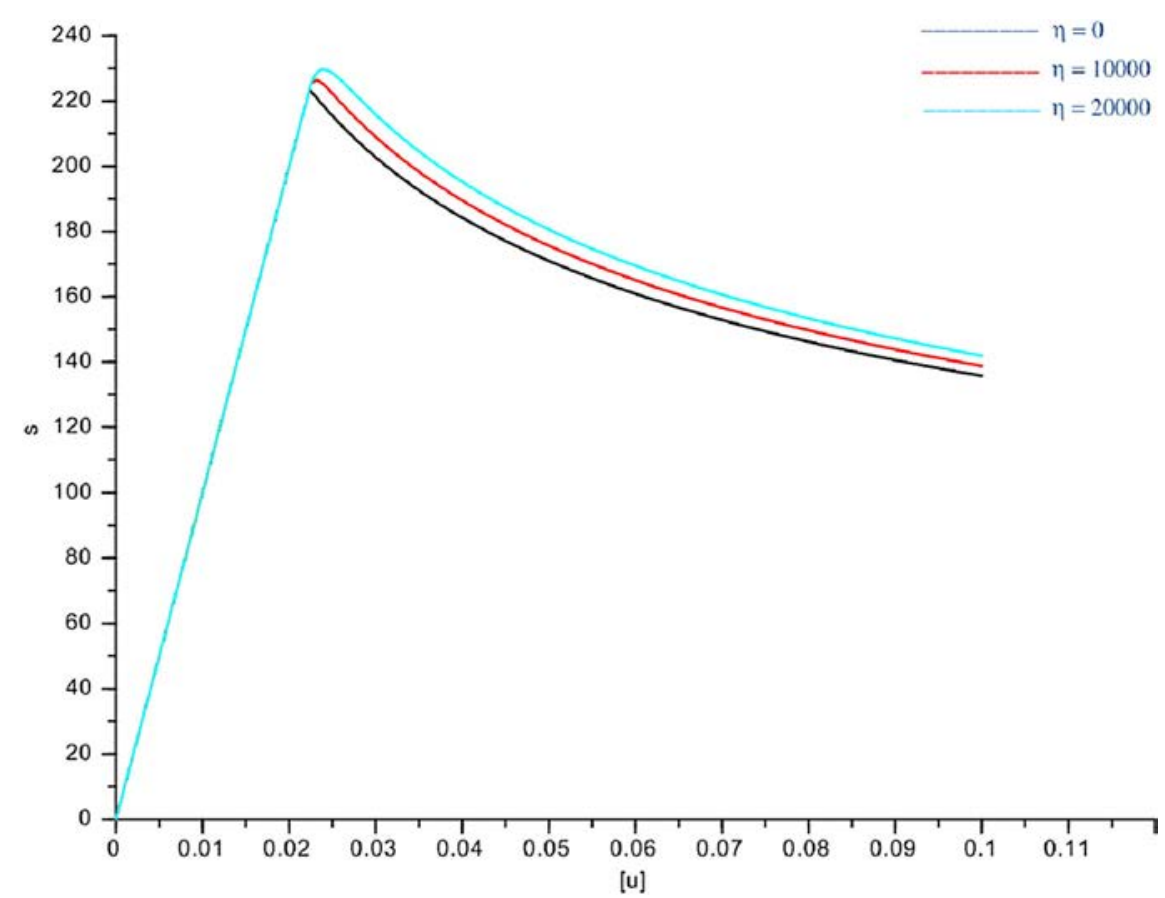

Fig. 2 Evolution of normal stress vs jump of displacement for various valors of viscosity (normalized values, $L=1, C=100, \omega=-50)$

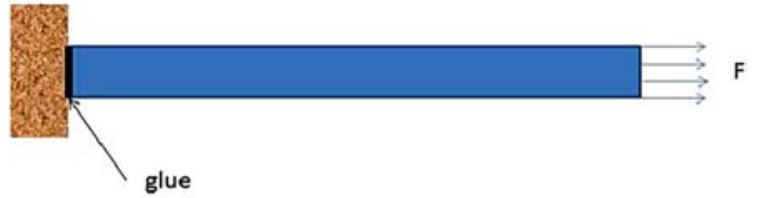

Fig. 3 A simple example

$$
F(t)= \begin{cases}F_{0} t & t \leq t_{1} \\ F_{0} t_{1} & t_{1} \leq t \leq t_{2} \\ \frac{F_{0} t_{2}}{t_{2}-t_{3}} t-\frac{F_{0} t_{2}}{t_{3}\left(t_{2}-t_{3}\right)} & t_{2} \leq t \leq t_{3}\end{cases}
$$

where $F_{0}$ and $t_{i}, i=1,2,3$ are given. It is obvious to show that the displacement field $u$ takes the form

$u(x)=\frac{F(t)}{E} x+u_{0}$,

where $E$ is the Young's modulus of the bar and $u_{0}$ is given by the interface law $\sigma=F(t)=\frac{C}{l^{2}} u_{0}$. The length $l$ is given by the equation

$b \dot{l}=\left(w+\frac{C}{l^{3}} u_{0}^{2}\right)_{+}$.
We take $l_{0}$ to denote the initial length of the crack. If $t \leq \sqrt{-\frac{\omega C}{l_{0}}}$, then $l=l_{0}$. If $t \geq \sqrt{-\frac{\omega C}{l_{0}}}$, then the crack length can be computed by a simple implicit Euler schema, i.e.

$$
l^{k+1}=\frac{l^{k}-\omega \frac{\Delta t}{b}}{1-\frac{\Delta t(F(t))^{2}}{b C}}
$$

where $\Delta t$ is taken to denote the time step, $l^{k}=l\left(t^{k}\right)$ and $t^{k}=k \Delta t$.

We can observe in Fig. 4 the increase of the crack length along time and in Fig. 5 the decrease of the stiffness of the glue in relation with the increase of the crack length for academic values of the coefficients. The model proposed here seems qualitatively coherent. During the first part of the loading (linear increase) and if $t<0.21$, the crack length remains constant $\left(l=l_{0}\right)$. When $t>0.21$ the crack length increases. We can observe the change of curvature corresponding to the variation of the loading. On fig. 5, we can see that the stiffness reduces to $1 / 25$ of its initial value during the process. 


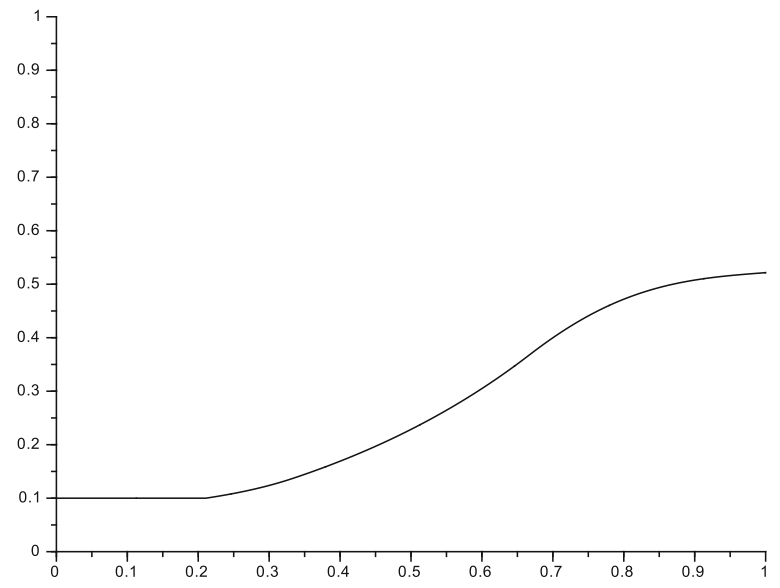

Fig. 4 Evolution of the crack length along time $\left(C=1, \omega=1, b=1, F_{0}=15, l_{0}=0.1, t_{3}=1, t_{1}=t_{3} / 3, t_{2}=2 t_{3} / 3\right)$

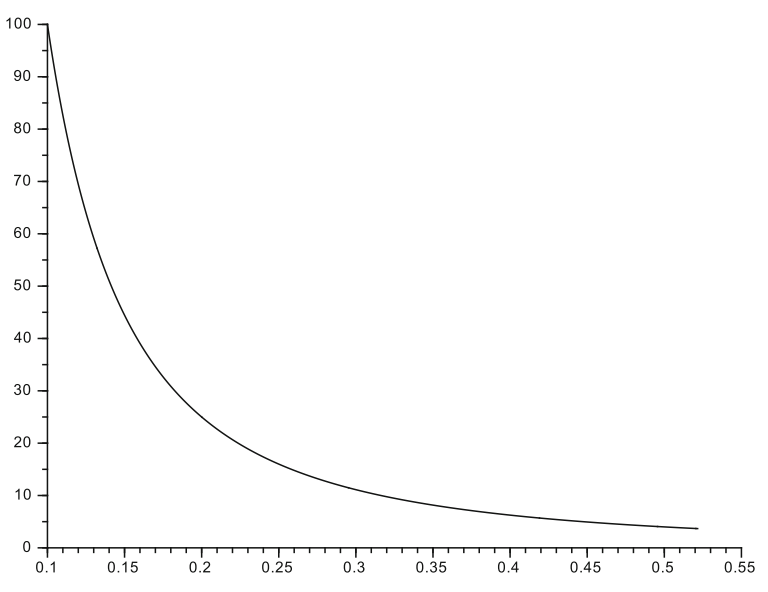

Fig. 5 Evolution of the stiffness vs crack length $C=1, \omega=$ $\left.1, b=1, F_{0}=15, l_{0}=0.1, t_{3}=1, t_{1}=t_{3} / 3, t_{2}=2 t_{3} / 3\right)$

\section{Conclusion}

In the first part of this paper, a model of damaged material has been proposed. This model is based on homogenization techniques and thermodynamics principles. The damage was governed by the evolution of the crack length at the micro-scale. In a second part of the paper, a model of imperfect interface has been derived from the asymptotic study of a three phases composite with perfectly bonding conditions between two adherents and an adhesive having the damaging behavior studied in the first part of the paper. The asymptotic expansion at the first order yields a model of imperfect interface taking into account damage. This argument can be also considered as a formal justification of well-known models for contact with adhesion (and unilateral conditions) which are deeply studied in the literature. The resulting model is studied in a simple example in in the one dimentional setting to show that the proposed model is qualitatively efficient.

In the future, the model of imperfect interface proposed in the present paper will be mathematically analyzed and numerically implemented to test its reliability and efficiency in more complex settings. Other kind of cracked materials [38] and damage evolutions models will also be studied. Stochastic processes could be also introduced in order to take into account the observed experimental variability of crack lengths .

Acknowledgments This research was partially supported by "Partenariats Hubert Curien (PHC)", Galilée Program 2015, Project number $32288 \mathrm{WH}$.

\section{References}

1. Benveniste Y, Miloh T (2001) Imperfect soft and stiff interfaces in two-dimensional elasticity. Mech Mater 33(6):309-323

2. Bonetti E, Bonfanti G, Rossi R (2008) Global existence for a contact problem with adhesion. Math Meth Appl Sci 31:1029-1064

3. Bonetti E, Bonfanti G, Rossi R (2007) Well-posedness and long-time behaviour for a model of contact with adhesion. Indiana Univ Math J 56:2787-2819

4. Bonetti E, Bonfanti G, Rossi R (2009) Thermal effects in adhesive contact: modelling and analysis. Nonlinearity 22:2697-2731 
5. Bonetti E, Bonfanti G, Rossi R (2011) Long-time behaviour of a thermomechanical model for adhesive contact. Discrete Contin Dyn Syst Ser S 4:273-309

6. Bonetti E, Bonfanti G, Rossi R (2012) Analysis of a unilateral contact problem taking into account adhesion and friction. J Differ Equ 253:438-462

7. Bonetti E, Bonfanti G, Rossi R (2014) Analysis of a temperature-dependent model for adhesive contact with friction. Physica D 285:42-62

8. Bonetti E, Bonfanti G, Rossi R (2015) Modeling via the internal energy balance and analysis of adhesive contact with friction in thermoviscoelasticity. Nonlinear Anal Real World Appl 22:473-507

9. Bornert PG, Bretheau T, Gilormini P (2001) Homogénéisation en mécanique des matériaux, Tome 1: Matériaux aléatoires élastiques et milieux périodiques. Hermes Sciences, Paris

10. Bouchitt G, Mielke A, Roubek T (2009) A complete-damage problem at small strains. Zeitschrift fur Angewandte Mathematik und Physik 60(2):205-236

11. Ciarlet PG (1988) Mathematical elasticity. volume I: threedimensional elasticity, vol I. North-Holland, Amsterdam

12. Challamel N, Girhammar UA (2011) Boundary-layer effect in composite beams with interlayer slip. J Aerospace Eng 24(2):199-209

13. Dumont S, Lebon F, Rizzoni R (2014) An asymptotic approach to the adhesion of thin stiff films. Mech Res Com 58:24-35

14. Frémond M (1987) Adhérence des solides. Journal de Mécanique Théorique et Appliquée 6:383-407

15. Frémond M (2001) Non-smooth thermo-mechanics. Springer, Berlin

16. Frémond M, Nedjar B (1996) Damage, gradient of damage and priciple of virtual power. Internat $\mathrm{J}$ Solids Struct 33:1083-1103

17. Fouchal F, Lebon F, Titeux I (2009) Contribution to the modelling of interfaces in masonry construction. Const Buld Mat 23:2428-2441

18. Fouchal F, Lebon F, Raffa ML, Vairo G (2014) An interface model including cracks and roughness applied to masonry. Open Civ Eng J 8:263-271

19. Geymonat G, Krasucki F, Lenci S (1999) Mathematical analysis of a bonded joint with a soft thin adhesive. Math Mech Sol 4(2):201-225

20. Hashin Z (2002) Thin interphase/imperfect interface in elasticity with application to coated fiber composites. J Mech Phys Solids 50(12):2509-2537

21. Ivanov IV, Velchev DS, Kneć M, Sadowski T (2011) Computational models of laminated glass plate under transverse static loading. In: Altenbach H, Eremeyev VA (eds) Shell-line structures, Non-classical theories and applications, Adv Struct Mat Springer, Berlin, pp 469-490

22. Klarbring A (1991) Derivation of the adhesively bonded joints by the asymptotic expansion method. Int J Eng Sci 29:493-512

23. Lebon F, Ould Khaoua A, Licht C (1998) Numerical study of soft adhesively bonded joints in finite elasticity. Comp Mech 21:134-140

24. Lebon F, Rizzoni R (2008) Asymptotic study on a soft thin layer: the non-convex case. Mech Adv Mat Struct 15:12-20
25. Lebon F, Rizzoni R (2010) Asymptotic analysis of a thin interface: the case involving similar rigidity. Int J Eng Sci 48:473-486

26. Lebon F, Rizzoni R (2011) Asymptotic behavior of a hard thin linear elastic interphase: an energy approach. Int J Sol Struct 48:441-449

27. Lebon F, Rizzoni R (2013) Modeling a hard, thin curvilinear interface. Discr Cont Dyn Syst Series S 6(6):1569-1586

28. Lebon F, Rizzoni R, Ronel-Idrissi S (2004) Asymptotic analysis of some non-linear soft thin layers. Comp Struct 82:1929-1938 -486

29. Lebon F, Ronel-Idrissi S (2004) Asymptotic analysis of Drucker-Prager and Mohr-Coulomb soft thin interfaces. Steel Comp Struct 4:133-147

30. Lebon F, Zaittouni S (2010) Asymptotic modelling of interfaces taking contact conditions into account: Asymptotic expansions and numerical implementation. Int J Eng Sci 48:111-127

31. Lenci S, Clementi F, Warminski J (2015) Nonlinear free dynamics of a two-layer composite beam with different boundary conditions. Meccanica 50(3):675-688

32. Li YD, Xiong T, Dong L (2015) A new interfacial imperfection coupling model (IICM) and its effect on the facture behavior of a layered multiferroic composite: Anti-plane case. Eur J Mech - A/Solids 52:26-36

33. Lopez-Realpozo JC, Rodriguez-Ramos R, Guinovart-Diaz R, Bravo-Castillero J, Otero JA, Sabina FJ, Lebon F, Dumont S, Sevostianov I (2014) Effective elastic shear stiffness of a periodic fibrous composite with non-uniform imperfect contact between the matrix and the fibers. Int $\mathrm{J}$ Solids Struct 51(6):1253-1262

34. Mauge C, Kachanov M (1994) Effective elastic properties of an anisotropic material with arbitrarily oriented interacting cracks. J Mech Phys Solids 42:561-584

35. Mielke A, Roubíček T, Thomas M (2012) From damage to delamination in nonlinearly elastic materials at small strains. J Elast 109(2):235-273

36. Nairn JA (2007) Numerical implementation of imperfect interfaces. Comp Mat Sci 40:525-536

37. Pelissou C, Lebon F (2009) Asymptotic modeling of quasibrittle interfaces. Comp Struct 87:1216-1223

38. Ponte Castaneda M, Willis J (1995) The effect of spatial distribution on the effective behavior of composite materials and cracked media. J Mech Phys Sol 43:1919-1951

39. Fouchal F, Lebon F, Raffa ML, Vairo G (2014) An interface model including cracks and roughness applied to masonry. Open Civil Eng J 8(1):263-271

40. Rekik A, Lebon F (2010) Identification of the representative crack length evolution in a multi-level interface model for quasi-brittle masonry. Int J Solids Struct 47(22-23):3011-3021

41. Rekik A, Lebon F (2012) Homogenization methods for interface modeling in damaged masonry. Adv Eng Softw 46(1):35-42

42. Rizzoni R, Lebon F (2012) Asymptotic analysis of an adhesive joint with mismatch strain. Eur J Mech A/Sol 36:1-8

43. Rizzoni R, Lebon F (2013) Imperfect interfaces as asymptotic models of thin curved elastic adhesive interphases. Mech Res Comm 51:39-50 
44. Rizzoni R, Dumont S, Lebon F (2015) On Saint Venant Kirchhoff Imperfect Interfaces., preprint

45. Rizzoni R, Dumont S, Lebon F, Sacco S (2014) Higher order model for soft and hard interfaces. Int J Solids Struct 51:4137-4148

46. Serpilli M (2014) Asymptotic analysis of a multimaterial with a thin piezoelectric interphase. Meccanica 49(7):1641-1652

47. Sevostianov I, Kachanov M (2014) On some controversial issues in effective field approaches to the problem of the overall elastic properties. Mech Mater 69:93-105
48. Sevostianov I, Kovik J, Siman-k F (2006) Elastic and electric properties of closed-cell aluminum foams: cross property connection. Mater Sci Eng A 420(1-2):87-99

49. Tsukrov I, Kachanov M (2000) Effective moduli of an anisotropic material with elliptical holes of arbitrary orientational distribution. Int J Solids Struct 37(41):5919-5941 